\title{
Hybrid
}

Revue des arts et médiations humaines

\section{Roman québécois et textualités numériques : transposition, amplification, parodie}

Sophie Marcotte

\section{OpenEdition}

10 Journals

Édition électronique

URL : https://journals.openedition.org/hybrid/342

DOI : $10.4000 /$ hybrid.342

ISSN : 2276-3538

Traduction(s) :

Digital textuality and the Quebecois novel: transposition, amplification, parody - URL : https:// journals.openedition.org/hybrid/426 [en]

Éditeur

Presses universitaires de Vincennes

Référence électronique

Sophie Marcotte, «Roman québécois et textualités numériques : transposition, amplification, parodie », Hybrid [En ligne], 5 | 2018, mis en ligne le 18 décembre 2018, consulté le 13 avril 2022. URL: http://journals.openedition.org/hybrid/342 ; DOI : https://doi.org/10.4000/hybrid.342

Ce document a été généré automatiquement le 13 avril 2022.

Revue Hybrid 


\title{
Roman québécois et textualités numériques : transposition, amplification, parodie
}

\author{
Sophie Marcotte
}

1 Les formes textuelles que les dispositifs numériques sans fil permettent d'échanger occupent une place de plus en plus importante dans l'imaginaire contemporain; elles envahissent, depuis plusieurs années déjà, le cinéma, le théâtre et la littérature. On trouve d'ailleurs beaucoup de traces de cette tendance dans la production littéraire québécoise de la dernière décennie. En effet, l'on voit transposés, dans la trame narrative des romans et nouvelles, des courriels, textos, statuts et commentaires Facebook, tweets ou entrées de blogs. En outre, les contacts des protagonistes avec leur entourage et avec le reste du monde reposent, dans plusieurs des cas, sur l'emploi systématique du téléphone portable, de la tablette ou de l'ordinateur (amplification). À certaines occasions, par ailleurs, le Web devient l'espace d'un récit de voyage virtuel, vécu à travers un écran d'ordinateur ou alimenté par des renseignements qui en ont été tirés.

2 Comme nous l'avons montré ailleurs, cette ouverture littéraire aux textualités numériques se manifeste de différentes façons. Au plan formel, d'abord, les fragments de textes numériques (réseaux sociaux, blogs, courriels, etc.) sont parfois insérés dans le récit de manière à alimenter les tensions et à le faire progresser jusqu'à son point culminant. C'est le cas, entre autres, dans les deux derniers thrillers du romancier québécois Jean-Jacques Pelletier : Dix petits hommes blancs (2014) et Machine God (2015) ${ }^{1}$ que nous avons présentés lors d'un colloque sur les narrations contemporaines, à Montréal, au printemps 2016². Au niveau du contenu, ensuite, l'apparition des textualités numériques paraît contribuer à l'élaboration de nouvelles représentations de la sociabilité dans la fiction et éclaire la manière dont se forment les liens entre les personnages, tout en concourant à mieux les définir. On pense notamment aux romans par courriel, encore peu nombreux dans le corpus québécois ${ }^{3}$, mais qui se multiplient aux États-Unis et en Europe ${ }^{4}$, aux romans appartenant à la catégorie de la chick lit, qui 
mettent en scène des personnages féminins à la vie amoureuse turbulente ${ }^{5}$, et aux journaux intimes virtuels, comme A Visit from the Goon Squad de Jennifer Egan, dont tout un chapitre est constitué du journal d'une jeune fille de 12 ans rédigé sous la forme d'une présentation PowerPoint, avec diagrammes et schémas à l'appui, et où le support numérique devient un outil pour l'aider à raconter son histoire'

\section{Un récit de voyage virtuel}

3 Or, il existe un autre cas de figure, illustré par le roman Document 1 de François Blais, paru en 2012 aux éditions L'instant même ${ }^{7}$. Dans Document 1, ce ne sont pas des fragments de texte associés à des appareils de communication numérique qui font progresser l'intrigue, comme on l'observe dans le roman d'espionnage, par exemple, et les personnages ne communiquent pas entre eux exclusivement par l'intermédiaire de plateformes d'échange virtuel, comme dans les plus récentes variantes du roman épistolaire. C'est plutôt le Web qui devient un espace de voyage. Le récit, comme on le verra, n'est dès lors pas celui d'un trajet réellement parcouru : il est plutôt celui d'un périple virtuel, Google permettant potentiellement l'accès au monde entier depuis un écran d'ordinateur.

4 Le roman de Blais raconte ainsi un voyage qui n'aura jamais lieu, de Grand-Mère, en Mauricie, à Bird-in-Hand, dans le comté de Lancaster, en Pennsylvanie. Cette destination a été déterminée au hasard par les protagonistes, Tess et Jude, qui ont parcouru des listes de noms étranges de villes américaines et les ont estimées toutes d'intérêt équivalent : «Il en va des villes américaines comme des épisodes de Virginie. $\mathrm{Tu}$ en as vu une, tu les as toutes vues ${ }^{8}$. Afin de financer leur périple, le duo commence à rédiger le récit du voyage à venir, que le logiciel Word enregistre automatiquement sous le titre "document 1 ». Ils espèrent que les recettes que celui-ci leur vaudra une fois publié leur permettront de défrayer ensuite les coûts du voyage réel : «On a calculé qu'on avait besoin de dix à quinze mille dollars [...] pour financer notre voyage ${ }^{9}$ ", écrit Tess, qui assure la narration de trente des trente-deux chapitres du récit - les deux autres étant pris en charge par son compagnon. Cette estimation des coûts inclut les frais de subsistance, l'achat d'une voiture usagée et le montant à consacrer à l'essence. Tess profite de la bonté de Sébastien Daoust, un docteur en littérature ayant publié un roman intitulé La Mort du ptérodactyle, qui travaille désormais dans une usine de fabrication de coques de bateaux. Celui-ci accepte d'apposer son nom sur une demande de subvention destinée au Conseil des arts du Canada puisque Tess et Jude n'y sont pas éligibles (ils n'ont jamais publié de roman); Daoust avance même aux deux grands voyageurs les douze mille dollars qui y sont réclamés. La suite du récit est quelque peu prévisible: Tess et Jude reçoivent finalement la subvention espérée et dépensent l'argent avant même d'avoir quitté Grand-Mère, découvrant la région dans leur rutilante Chevrolet Monte Carlo et s'offrant des repas hors de prix dans des restaurants de haute renommée. Le voyage à Bird-in-Hand n'aura jamais lieu; les protagonistes ne dépasseront jamais d'autres frontières que celles du virtuel.

\section{La quête virtuelle du bonheur}

On pourrait très certainement voir, dans Document 1, un écho à la forme du road novel, qui a d'ailleurs connu un regain d'intérêt chez les romanciers québécois au cours des 
dix ou quinze dernières années. On peut penser, entre autres, aux deux romans de Guillaume Vigneault, Carnets de naufrage et Chercher le vent - clairement inspirés de Volkswagen Blues de Jacques Poulin et du mythique On the Road de Jack Kerouac -, à La Foi du braconnier de Marc Séguin, à Sur la 132 de Gabriel Anctil et au Fil des kilomètres de Christian Guay-Poliquin, qui reposent tous sur la quête individuelle d'un protagoniste masculin qui cherche à redonner un sens à son existence en voyageant à travers le continent nord-américain ${ }^{10}$.

Or, François Blais propose plutôt une parodie du récit de voyage. D'abord, il ne met pas le personnage masculin au cœur de la "quête ", mais bien un couple, ou plutôt un duo improbable formé d'une jeune fille préparant des sous-marins chez Subway, et d'un prestataire d'aide sociale, qui partagent un appartement situé dans le centre-ville de Grand-Mère. La fille, qui assure presque l'entièreté de la narration, est très clairement le pôle dominant du couple. Ensuite, les protagonistes ne partent pas à l'aventure sans connaître leur trajet ou leur destination finale : ils se rendent plutôt « sur Google Maps pour choisir une destination et convenir d'un itinéraire ${ }^{11}[\ldots] »$... La finalité du voyage n'est dès lors pas clairement établie. En effet, si l'une des prémisses du roman de la route est la quête d'une forme de bonheur et de rédemption, dans Document 1, on a plutôt affaire à un couple de losers qui se croient malheureux, mais qui en même temps restent très lucides, se permettant même de se moquer de tous les malheureux de ce monde (un clin d'œil, peut-être, à tous ceux qui adhèrent aveuglément aux discours de psycho-pop qui abondent sur le Web et sur les réseaux sociaux). On peut lire, à cet effet, dans l'incipit du roman :

C'est épais, du monde malheureux, ça pense que ça existe pour vrai, changer le mal de place, ça s'imagine toujours que le bonheur est ailleurs, ça veut prendre de nouveaux départs, remettre le compteur à zéro, partir pour mieux se retrouver et toutes ces niaiseries-là ${ }^{12}$.

Jude prétend, de toute façon, que Tess et lui sont «trop insignifiants pour être malheureux ${ }^{13}$ ».

7 L'errance (ou la navigation) sur le Web pourrait tout de même rappeler, à certains égards, la dérive des personnages du roman de la route :

Jude et moi [...], on aimait bien, également, errer sans but à travers le monde surtout à travers l'Amérique, en fait, cela pour des raisons qui seront expliquées plus loin - grâce à Google Earth, Google Maps et Bing Maps. On pouvait, par exemple, faire le tour de la Gaspésie en vingt minutes ${ }^{14}[. .$.$] .$

8 La multiplication de ces déplacements virtuels, qui deviendra la base du roman racontant d'avance le voyage à Bird-in-Hand, est une manière de se moquer de tous ceux qui publient de tels récits. En anticipant le refus éventuel des éditeurs (et en prenant soin d'écorcher, au passage, certaines maisons d'édition qui ne font pas beaucoup de place à la relève), Tess s'empresse de préciser que "plus personne ne publie de récit de voyage de nos jours ${ }^{15}$ ». Il s'agit d'« un genre moribond», titre donné à un chapitre de Document 1 dans lequel il est question de récits de voyage de Marco Polo, Cortez, Cook, Bougainville, Stendhal, Tocqueville, beaucoup plus grandioses que celui des deux comparses de Shawinigan. Le Web rend désormais toute forme de déplacement inutile, puisqu'

aujourd'hui tout le monde peut aller partout ou, à défaut, tout le monde peut mémérer ce qui se trame à Rio de Janeiro ou à Fort Myers, et chacun peut savoir, pourvu que cela l'intéresse, que Jason Parrish Casebier, domicilié au 2219, Florence Boulevard, à Omaha (Nebraska), a été condamné pour "rape felony» le 25 novembre $1995^{16}$. 
Surtout, le Web permet d'être à la fois n'importe où - dont à Oklahoma City - et nulle part - soit dans son salon :

Pourtant, on ne se tannait pas, on restait jusqu'à pas d'heure devant l'ordi à sillonner les rues d'Edmond ou à espionner le centre-ville d'Oklahoma City grâce à la Web cam placée sur le toit de l'hôtel de ville, s'extasiant chaque fois qu'un badaud passait dans le cadre ${ }^{17}$.

\section{Un trop-plein d'informations}

10 Ce regard moqueur sur le récit de voyage traditionnel, perverti en voyage virtuel parce qu'on a accès, sur le Web, à tous les renseignements nécessaires pour l'entreprendre, vient aussi mettre en évidence une autre problématique liée au Web 2.0, celle du « tropplein d'informations » auquel il donne accès - une problématique que les protagonistes de Document 1 illustrent à merveille. En effet, ceux-ci montrent bien la manière dont une certaine part de la population interagit avec cette "overdose d'informations ${ }^{18}$ ». Internet, comme le soulignait déjà Alexandre Serres en 2005, est devenu « le nouveau régime de vérité, notamment pour les jeunes générations, qui y puisent une large part de leurs informations sur l'actualité et sur le monde ${ }^{19} »$. Tout, dans le roman de Blais, se moque allègrement de ce phénomène.

11 On peut évidemment citer l'exemple de Wikipédia, qui sert de source primaire de renseignements sur les États-Unis au duo de voyageurs. Blais, qui a d'ailleurs lui-même admis, dans un entretien accordé au Devoir, que le Web est « un peu son interface avec le monde ${ }^{20}$ ", a recopié intégralement des articles provenant du site de l'encyclopédie en ligne, accomplissant, de ce fait, le même geste que des millions de personnes dont les recherches reposent exclusivement sur les informations trouvées sur ce site chaque jour. Son personnage explique ainsi le processus: «On allait ensuite demander à Wikipédia tout ce qu'il y avait à savoir sur l'endroit. La plupart du temps, ça se résumait à pas grand-chose [...], mais quelquefois on avait droit à de jolies histoires ${ }^{21}$. " Parmi ces "jolies histoires", on note la transcription de l'article qui explique les raisons pour lesquelles Hot Springs au Mexique a changé son nom pour Truth or Consequences au début des années 1950... Les personnages de Document 1 trouvent même le moyen d'identifier des lacunes au savoir proposé par Wikipédia, à commencer par le fait qu'il n'y a pas d'article sur la ville de Knob Lick, alors qu'il s'en trouve sur « Le problème de la sexualité entre les hommes et les sirènes dans la littérature »; [...] «Liste des personnages de fictions avec neuf doigts »; [...] «La mucophagie» (ou consommation de mucus); [...] «Nils Olav, le pingouin colonel en chef dans la garde royale norvégienne »; "Liste des cochons historiques»; [...] «La religion en Antarctique $^{22}[. .$.$] ». En outre, « on apprend au passage que les bornes-fontaines sont$ jaunes à Des Moines (Iowa) ${ }^{23}$ ». Le Web, autrement dit, regorge de renseignements absolument inutiles, qui attirent l'attention de ceux qui se trouvent incapables de hiérarchiser les informations selon leur importance. Tout devient, comme le rappelle la narratrice, « un renseignement crucial de plus à occuper dans l'espace de stockage de nos cerveaux ${ }^{24} »$. Au-delà du fait qu'il sous-entende que l'époque contemporaine tend à favoriser la superficialité et l'absence d'esprit d'analyse, le roman de Blais illustre bien, pour aller même un peu plus loin, «dans quelle mesure (un) [...] trop-plein d'informations peut (éventuellement) conduire à une régression $[. . .]^{25} »$.

Hybrid, 5 | 2018 

paru en $2014^{26}$, qui détourne non pas la forme traditionnelle du road novel, mais celle du journal intime. Sam met en scène une narratrice sans ambition, comme les personnages de Document 1 ; il propose le journal personnel (fictif, on le comprend à la fin du roman) de cette jeune femme qui mène une existence banale et qui évite surtout toute forme de vie sociale. Sam raconte, dans son journal, son exploration des villages voisins sur le Web, villages qu'elle va ensuite visiter ; elle précise passer son temps libre à flâner sur Internet et recopie les moindres informations qu'elle peut y trouver - elle retranscrit notamment une liste de sites pornographiques de neuf pages $^{27}-$, ce qui a pour effet d'amplifier, comme dans Document 1, l'aspect superficiel et anecdotique du Web :

[...] j'ai ouvert l'ordi et je suis allée vagabonder un peu dans Internet, où j'ai essayé de partir quelques chicanes sur des blogs de mongols et fait quelques demandes d'amitié à des Indonésiennes qui s'appellent Caca, c'est un nom assez commun làbas, $[. .$.$] le pire c'est que je me trouve drôle \mathrm{l}^{28}[. .$.$] .$

\section{Une critique de la médiocrité}

Le trop-plein d'informations dans lequel nagent les deux protagonistes de Document 1 s'accompagne d'une critique du Web à un second niveau: celui-ci regorge non seulement de renseignements plus ou moins justes et utiles au commun des mortels, mais pose un regard très critique sur son contenu littéraire, en se moquant du fait que des œuvres littéraires médiocres y trouvent leur place. Tel qu'évoqué précédemment, et comme le soutient Dominique Monet dans un ouvrage consacré au multimédia, la société contemporaine « exclurait toute rigueur intellectuelle et tout esprit d'analyse ». Elle mettrait en évidence une incapacité chez l'individu «à trier, comprendre, digérer puis transformer la masse des informations qui n'apprennent rien par elles-mêmes, qui ne sont en fait que des données brutes à analyser et qui nous submergent ${ }^{29}$. La digression sur des auteurs obscurs de la région de la Mauricie souligne cette nouvelle réalité à grands traits. En effet, après une longue description biobibliographique de Paule Doyon, une auteure originaire de Grand-Mère - il ne faut pas mourir sans l'avoir lue, car « c'est très mal vu dans l'au-delà ${ }^{30}$ »-, Tess s'empresse de révéler « un secret » au lecteur concernant les romans médiocres de l'écrivaine : « une partie importante de l'œuvre de Paule Doyon t'est accessible sans que tu aies à bouger ton derrière ni dépenser un seul kopeck: en te rendant sur son site (http://www.cafe.rapidus.net/ anddoyon/index.html ${ }^{31}$ ». De la même manière, la narratrice se moque allègrement de Bryan Perro, l'auteur de la série pour la jeunesse Amos Daragon, et des Descôteaux, scénaristes du téléroman Entre chien et loup diffusé de 1984 à 1992, tous également originaires de la Mauricie, rappelant ainsi que «Grand-Mère compte parmi ses habitants un nombre impressionnant de plumes renommées ${ }^{32}$ ». Ces renseignements, qui n'ont absolument rien à voir avec l'intrigue principale, celle du voyage à Bird-inHand, viennent montrer encore à quel point il est facile de se perdre dans le trop-plein $\mathrm{du}$ Web, et combien celui-ci peut parfois laisser croire à des gens sans discernement que l'apport d'auteurs de troisième ordre est aussi important que celui d'autres de premier plan. «On appelle n'importe quoi "roman" de nos jours ${ }^{33}$ ", finit par avouer Tess.

14 Les déambulations des personnages sur la Toile finissent en outre par faire en sorte qu'ils se définissent à travers celles-ci. La narratrice, qui tente de respecter les règles établies par le gourou des lettres Marc Fisher, dans son célèbre ouvrage Conseil à un

Hybrid, 5 | 2018 
jeune romancier où il estime qu'il importe de « présenter les personnages petit à petit, au fil du récit ", ou de "ventiler l'information », pour le dire autrement, choisit plutôt de se présenter tout d'un bloc. Sa technique : trouver et remplir un test de personnalité sur le Web:

Qu'est-ce qu'on raconte une fois qu'on a dit son nom, son âge, son lieu de résidence et sa profession? [...] je devrais être en mesure de dénicher dans le Web un quelconque test de personnalités que je compléterai sous tes yeux. (site www.sedecouvrir.fr) ${ }^{34}$

Au bout du compte, même le grand Marc Fisher ne fait pas le poids par rapport à tous les conseils aux écrivains qu'il est possible de trouver par une simple recherche Google :

De toute façon, son enseignement ne pouvait m'être d'aucune utilité, n'ayant aucune intention d'écrire de la schnoute expérimentale. Je me suis plutôt tournée vers notre mentor à tous, Google, et je lui ai demandé sans détour : "Comment se faire publier ${ }^{35}$ ?»

Le roman de Blais rappelle que Google devient, d'une certaine manière, l'équivalent du narrateur-qui-sait-tout, de celui qui connaît les personnages encore mieux qu'euxmêmes; qu'il prévoit certains de leurs comportements, certains de leurs " déplacements "; qu'il leur fabrique même une identité de laquelle ils ne soupçonnent pas eux-mêmes toutes les ramifications et les traces.

\section{Conclusion}

Que se passe-t-il, en somme, quand la fiction s'empare du numérique? Document 1 de François Blais, on vient de le voir, propose un récit de voyage nouveau genre, qui n'aura d'autres lieux que le canapé de l'appartement de Tess et Jude, à Grand-Mère, et l'écran d'ordinateur qui le projette. À quoi bon se déplacer, de toute manière, à l'ère du numérique? C'est l'une des questions que pose le roman, notamment à travers l'épisode de l'achat de la voiture usagée :

On s'est permis de lui faire remarquer (au père de Jude) qu'il était inutile - voire absurde - à notre époque de se transporter physiquement chez un vendeur de voitures. Chez son Grenier, à Saint-Étienne-des-Grès, on trouverait, quoi? une centaine de machines, à tout casser, alors que trois cent vingt-neuf mille véhicules sont répertoriés sur le site autohebdo.net ${ }^{36}$.

En ce sens, le roman propose une réflexion tout à fait actuelle sur les phénomènes liés à l'omniprésence du Web et des technologies de l'information et des communications dans le quotidien : l'instantanéité, l'incapacité de prendre du recul, de réfléchir, face à cette vague déferlante d'informations, l'accentuation, pour reprendre les mots de JeanPierre Balpe, "de la coupure" entre ceux et celles qui possèdent la capacité de discernement et d'autres "qui n'ont pu accéder à ce niveau »" ${ }^{37}$, et qui du coup sont incapables de distinguer la frontière entre l'important et le futile.

En définitive, Document 1 s'inscrit, de manière plus générale, dans cette récente tendance dans la littérature contemporaine à actualiser certaines formes de récits traditionnels en les parodiant par l'insertion dans le monde numérique. En effet, outre le récit de voyage dont se moque Blais, des romans par courriel se moquent du quotidien dans l'entreprise actuelle, à la manière de la série britannique The Office. Par ailleurs, le principe même de la consultation du Web, associé à la métaphore de la navigation, constitue une forme de voyage: ouvrir une nouvelle fenêtre, dans un navigateur web, devient l'équivalent d'un départ. C'est notamment le cas dans le roman 
de Guillaume Morissette, Nouvel onglet, où le narrateur, au terme de plusieurs mois d'une vie consacrée à la fête et à la consommation d'alcool et de drogue, estime qu'il est peut-être temps pour lui d'ouvrir cette "nouvelle fenêtre». Il ne s'agit plus d'un voyage virtuel par l'intermédiaire d'applications de localisation, comme dans Document 1 , on l'aura deviné, mais bien d'une volonté de refaire sa vie, peut-être à l'écart, justement, du Web et des médias socio-numériques, comme l'affirme le narrateur à la fin du récit qui, dans un improbable élan de lucidité, avoue même avoir "soudainement eu envie de plier Facebook et d'en faire un héron en origami ${ }^{38}$ ".

\section{BIBLIOGRAPHIE}

ANCTIL Gabriel, Sur la 132, Montréal, Héliotrope, 2013.

BLAIS François, Iphigénie en haute-ville, Québec, L’instant même, 2009.

BLAIS François, Document 1 [2012], Québec, L'instant même, 2013,

BLAIS François, Sam, Québec, L'instant même, 2014.

EGAN Jennifer, A Visit from the Goon Squad, Washington (D.C.), Anchor Books, 2011.

[s.a.], « Des effets de l'abondance d'information et de la distraction sur notre attention », Digital Society Forum. [En ligne] https://digital-society-forum.orange.com/fr/les-forums/

554-12._des_effets_de_labondance_dinformation_et_de_la_distraction_sur_notre_attention [consulté le 15 février 2018].

GERVAIS Bertrand, La Dernière guerre, Montréal, XYZ, 2017.

GuAY-POLIQUin Christian, Le Fil des kilomètres, Saguenay, La Peuplade, 2013.

KeROUAC Jack, On the Road [1957], Londres, Penguin Books, 2000.

LALONDE Catherine, «La nonchalance de François Blais », Le Devoir, $1^{\mathrm{er}}$ mars 2014. [En ligne]

http://www.ledevoir.com/lire/401308/la-nonchalance-de-francois-blais [consulté le

29 janvier 2018].

LÉVY Pierre et BALPE Jean-Pierre, « [entretien] », propos recueillis par Michel ABERGANTI, supplément, Le Monde, 20 novembre 1997. [En ligne] http://www.site-magister.com/bts/ synthese3b.htm\#axzz5ZU7FRQka [consulté le 5 février 2018].

MARCOTTE Sophie, «La sociabilité numérique comme ressort de la fiction », Sens public, décembre 2016. [En ligne] http://www.sens-public.org/article1225.html [consulté le 15 janvier 2018].

MARCoTtE Sophie, « Médias sociaux et narration romanesque chez Jean-Jacques Pelletier », communication présentée lors du colloque « Narrations contemporaines : écrans, médias et documents ", UQAM, avril 2016. [En ligne] http://oic.uqam.ca/fr/communications/ medias-sociaux-et-narration-romanesque-chez-jean-jacques-pelletier [consulté le 2 février 2018]. Millerand Florence, RUEFF Julien et ProulX Serge (dir.), Web social. Mutations de la communication, Québec, Presses de l'Université du Québec, 2010. 
MONET Dominique, Le Multimédia, Paris, Flammarion, 1998.

MORISSETTE Guillaume, Nouvel onglet, trad. Daniel Grenier, Montréal, Boréal, 2016.

Pelletier Jean-Jacques, Dix petits hommes blancs, Montréal, Hurtubise, 2014.

PelletieR Jean-Jacques, Machine God, Montréal, Hurtubise, 2015.

PouLiN Jacques, Volkswagen Blues [1984], Montréal, Leméac, 2015.

SÉGUIN Marc, La foi du braconnier, Montréal, Leméac Éditeur, 2009.

SERRES Alexandre, «Évaluation de l'information sur Internet. Le défi de la formation », 2005.

[En ligne] http://bbf.enssib.fr/consulter/bbf-2005-06-0038-006 [consulté le 15 février 2018].

VIGNEAULT Guillaume, Carnets de naufrage [2000], Montréal, Boréal, 2001.

VIGNEAULT Guillaume, Chercher le vent [2001], Montréal, Boréal, 2003.

\section{NOTES}

1. Jean-Jacques Pelletier, Dix petits hommes blancs, Montréal, Hurtubise, 2014 ; Id., Machine God, Montréal, Hurtubise, 2015.

2. Voir Sophie Marcotte, "Médias sociaux et narration romanesque chez Jean-Jacques Pelletier », communication présentée lors du colloque «Narrations contemporaines : écrans, médias et documents ", UQAM, 2016. [En ligne] http://oic.uqam.ca/fr/communications/medias-sociaux-etnarration-romanesque-chez-jean-jacques-pelletier [consulté le 2 février 2018].

3. Le roman Iphigénie en haute-ville de François Blais (Québec, instant même, 2009) est l'un des seuls exemples de roman québécois entièrement rédigé sous la forme de courriels.

4. Voir, entre autres, Maria Semple, Where'd you go, Bernadette (2012), New York/Boston/Londres, Little Brown and Company, 2014 ; Daniel Glattauer, Quand souffle le vent du nord, trad. Anne-Sophie Anglaret, Paris, Grasset, 2010 ; Martina Wachendorff, Le Baiser électrique, Paris, Gallimard, 2001 ; Matt Beaumont, E, Londres, Plume, 2000 ; Matt Beaumont, $E^{2}$ [2009], Londres, Plume, 2010.

5. Pour une étude des nouveaux modes de sociabilité introduits par le numérique dans ce type de romans, voir Sophie Marcotte, "La sociabilité numérique comme ressort de la fiction ", Sens public, décembre 2016. [En ligne] http://www.sens-public.org/article1225.html [consulté le 15 janvier 2018].

6. Jennifer Egan, A Visit from the Goon Squad, Washington (D.C.), Anchor Books, 2011.

7. Le roman a été réédité en format poche dès l'année suivante : François Blais, Document 1 [2012], Québec, L'instant même, 2013. Les citations tirées du roman renverront à cette seconde édition.

8. François Blais, Document 1, Québec, L'instant même, 2013, p. 18.

9. François Blais, Document 1, Québec, L'instant même, 2013, p. 40.

10. Guillaume Vigneault, Carnets de naufrage [2001] Montréal, Boréal, 2005 ; Guillaume Vigneault, Chercher le vent [2001], Montréal, Boréal, 2003 ; Jacques Poulin, Volkswagen Blues [1984], Montréal, Leméac, 2015 ; Jack Kerouac, On the Road [1957], Londres, Penguin Books, 2000 ; Marc Séguin, La Foi du braconnier, Montréal, Leméac, 2009; Gabriel Anctil, Sur la 132, Montréal, Héliotrope, 2013 ; Christian Guay-Poliquin, Le Fil des kilomètres, Saguenay, La Peuplade, 2013.

11. François Blais, Document 1, Québec, L'instant même, 2013, p. 139.

12. François Blais, Document 1, Québec, L'instant même, 2013, p. 9.

13. François Blais, Document 1, Québec, L'instant même, 2013, p. 74.

14. François Blais, Document 1, Québec, L'instant même, 2013, p. 16.

15. François Blais, Document 1, Québec, L'instant même, 2013, p. 62.

16. François Blais, Document 1, Québec, L'instant même, 2013, p. 64. 
17. François Blais, Document 1, Québec, L'instant même, 2013, p. 18.

18. Voir [s.a.]. « Des effets de l'abondance d'information et de la distraction sur notre attention ", Digital Society Forum. [En ligne] https://digital-society-forum.orange.com/fr/les-forums/ 554-12._des_effets_de_labondance_dinformation_et_de_la_distraction_sur_notre_attention dinformation_et_de_la_distraction_sur_notre_attention [consulté le 15 février 2018].

19. Alexandre Serres, «Évaluation de l'information sur Internet. Le défi de la formation », 2005. [En ligne] http://bbf.enssib.fr/consulter/bbf-2005-06-0038-006 [consulté le 15 février 2018].

20. Catherine Lalonde, «La nonchalance de François Blais » Le Devoir, $1^{\mathrm{er}}$ mars 2014. [En ligne] http://www.ledevoir.com/lire/401308/la-nonchalance-de-francois-blais [consulté le 29 janvier 2018].

21. François Blais, Document 1, Québec, L'instant même, 2013, p. 19.

22. François Blais, Document 1, Québec, L'instant même, 2013, p. 143.

23. François Blais, Document 1, Québec, L'instant même, 2013, p. 17.

24. François Blais, Document 1, Québec, L'instant même, 2013, p. 17.

25. Florence Millerand, Julien Rueff et Serge Proulx (dir.), Web social. Mutation de la communication, Québec, Presses de l'Université du Québec, 2010, p. 25.

26. François Blais, Sam, Québec, L'instant même, 2014, p. 191.

27. François Blais, Sam, Québec, L'instant même, 2014, p. 73-82.

28. François Blais, Sam, Québec, L'instant même, 2014, p. 72.

29. Dominique Monet, Le Multimédia, Paris, Flammarion, 1998, p. 58.

30. François Blais, Document 1, Québec, L'instant même, 2013, p. 49.

31. François Blais, Document 1, Québec, L'instant même, 2013, p. 50.

32. François Blais, Document 1, Québec, L'instant même, 2013, p. 49.

33. François Blais, Document 1, Québec, L'instant même, 2013, p. 65.

34. François Blais, Document 1, Québec, L'instant même, 2013, p. 28.

35. François Blais, Document 1, Québec, L'instant même, 2013, p. 84 sq.

36. François Blais, Document 1, Québec, L'instant même, 2013, p. 128 sq.

37. Pierre Lévy et Jean-Pierre Balpe, «[entretien]», propos recueillis par Michel Aberganti, supplément, Le Monde, 20 novembre 1997. [En ligne] http://www.site-magister.com/bts/ synthese3b.htm\#axzz5ZU7FRQka [consulté le 5 février 2018].

38. Guillaume Morissette, Nouvel onglet, trad. Daniel Grenier, Montréal, Boréal, 2016, p. 245.

\section{RÉSUMÉS}

Les formes textuelles que les dispositifs numériques sans fil permettent d'échanger occupent une place de plus en plus importante dans l'imaginaire contemporain. On trouve d'ailleurs beaucoup de traces de cette tendance dans la production littéraire québécoise. C'est le cas du roman Document 1 de François Blais, dans lequel, d'une part, les contacts des protagonistes avec le reste du monde reposent sur l'emploi de dispositifs numériques, et où, d'autre part, le Web devient l'espace d'un récit de voyage virtuel, vécu à travers un écran d'ordinateur. 
INDEX

Mots-clés : amplification, Google, parodie, récit de voyage, roman québécois, textualités numériques, transposition

\section{AUTEUR}

\section{SOPHIE MARCOTTE}

Sophie Marcotte est professeure titulaire de littérature au département d'études françaises de l'Université Concordia. Ses recherches les plus récentes portent sur les liens entre littérature et technologies. Elle a entre autres publié (avec Samuel Archibald, UQAM), L'Imaginaire littéraire du numérique (PUQ, 2015) et elle a codirigé, avec Sylvain David (Concordia), un dossier de la revue Sens public sur l'archive à l'ère du numérique (2016). Elle est la responsable du projet HyperRoy, qui consiste en l'édition électronique des manuscrits et des inédits de la romancière québécoise Gabrielle Roy. Site web : http://hyperroy.nt2.uqam.ca/. 